\title{
Diseño óptimo para transmisión de fuerza en un efector final
}

\author{
Eric Santiago-Valentín*, Adrián Solano-Palma, Pedro Bautista-Camino, \\ José Marco Antonio Rueda-Meléndez, Edgar Alfredo Portilla-Flores \\ Instituto Politécnico Nacional, \\ Centro de Innovación y Desarrollo Tecnológico en Cómputo, \\ México D.F., México \\ e.santiag.valentin@gmail.com, asolanop@ipn.mx, peterbc1@gmail.com, \\ jrueda@ipn.mx, aportilla@ipn.mx
}

\begin{abstract}
Resumen. En este trabajo se presenta una solución novedosa para el diseño óptimo de un efector final tipo pinza, para la transmisión de fuerza constante en su espacio de trabajo o agarre. Para tal fin se propone un problema de optimización numérica con restricciones asociado al mecanismo, el cual se resuelve utilizando el algoritmo de evolución diferencial. La función objetivo propuesta es la transmisión de fuerza constante por parte del eslabón de agarre. Los resultados obtenidos muestran una transferencia de fuerza constante con diferentes configuraciones del efector final, comprobándose la naturaleza multimodal del problema.
\end{abstract}

Palabras clave: efector final, evolución diferencial, optimización, restricciones, síntesis, transmision de fuerza.

\section{Introducción}

Actualmente, el proceso de diseño industrial obliga al ingeniero a utilizar metodologías alternas para obtener sistemas mejores y más eficientes. Una de éstas técnicas consiste en traducir el problema original de diseño en un problema de optimización numérica, con el propósito de obtener combinaciones válidas de valores para los parámetros que describen al sistema, buscando un desempeño óptimo del mismo. Sin embargo, en la mayoría de los casos se tienen problemas duros de optimización, cuya solución presenta una alta complejidad. En los últimos años los algoritmos evolutivos han tenido un gran auge, aplicándose exitosamente a la solución de problemas de ingeniería del mundo real. Un algoritmo líder dentro de esta clase de metodologías es el propuesto por Rainer Storn en 1995 [2], denominado Evolución Diferencial (ED); desde entonces su eficacia ha sido puesto a prueba una y otra vez, demostrando su utilidad en problemas de optimización que en muchas ocasiones no pueden resolverse por métodos clásicos y/o presentan una alta dificultad de implementación.

\footnotetext{
* Autor para correspondencia.
} 
La optimización en aplicaciones industriales y tecnologicas tiene una alta importancia, debido a la disminución potencial de costos que se puede obtener, que va desde el ahorro de material hasta la reducción de los tiempos de ejecución. Los efectores finales tipo pinza (grippers) de dos dedos, cuya función principal es la manipulación de objetos, se utilizan ampliamente en automatización [1]. En el proceso de diseño de estos elementos es importante considerar aspectos tales como el tipo y las dimensiones del objeto a manipular, o el peso final del efector y su tipo de accionamiento (eléctrico o neumático), por mencionar algunos; todo ello con el fin de obtener una interfaz adecuada entre el sistema automatizado, el espacio de trabajo del efector final y el objeto de interés.

El diseño óptimo de grippers ha sido abordado desde diferentes perspectivas. En [3] se propone un algoritmo génetico para diseñar un efector final robótico, mientras que Cuadrado et al. [4] desarrollaron una solución para grippers de dos dedos, aplicando una función nativa de Matlab®. Saravanan et al. [5] presentaron un estudio para el diseño óptimo de este elemento por medio de tres diferentes algoritmos evolutivos, planteándolo como un problema multi-objetivo. En [9] se hizo la optimización de dos efectores finales comerciales mediante un problema multi-objetivo utilizando el toolbox de Matlabß. En [6] se plantea el problema como multi-objetivo, resolviéndolo mediante el algortimo de colonia artificial de hormigas, obteniendo una alta precisión en los resultados. Portilla et al. [7] resolvieron el caso de un efector final de tres dedos utilizando el algoritmo de forrajeo de bacterias, planteando el problema como mono-objetivo; de igual forma, en [8] se propuso un problema de optimización de un gripper para una planta embotelladora, utilizando ED pero aplicándolo únicamente a una de las piezas mecánicas del sistema y no a su totalidad.

En este trabajo se presenta una solución novedosa al problema de síntesis dimensional de un efector final tipo pinza, para la transmisión de fuerza constante en su espacio de trabajo o agarre, utilizando el algoritmo de ED. La organización del artículo es la siguiente: en la Sección 2 se plantea el problema y se analiza el mecanismo para la obtención de las ecuaciones que describen al sistema; en la Sección 3 se detalla la estrategia de optmización aplicada, basada en el algortimo de ED, mientras que en la Sección 4 se definen los parámetros del sistema para su optimización. En la Sección 5 se describe el algoritmo de ED así como su implementación computacional; en la Sección 6 se revisan los resultados obtenidos y, finalmente, en la Sección 7 se presentan las conclusiones.

\section{Problema de síntesis del efector final}

Sea el efector final tipo pinza que se muestra en la Figura 1, integrado por los siguientes elementos: pieza base (1), tornillo de potencia y tuerca (2), eslabón de agarre (3) y eslabón de acoplamiento (4). El principio operacional del efector es el siguiente: fijando un sistema coordenado de referencia en la pieza base, la posición mínima de agarre ocurre cuando el desplazamiento de la tuerca es cercano a cero, es decir, cuando la posición relativa del elemento 2 es mínima respecto del origen de dicho sistema coordenado; así mismo, la máxima posición 
se obtiene cuando la distancia entre la tuerca y el sistema de referencia es máxima. Una vez satisfecha la condición del rango de apertura del efector, se debe asegurar en dicho rango una fuerza de agarre constante sobre el objeto de interés.

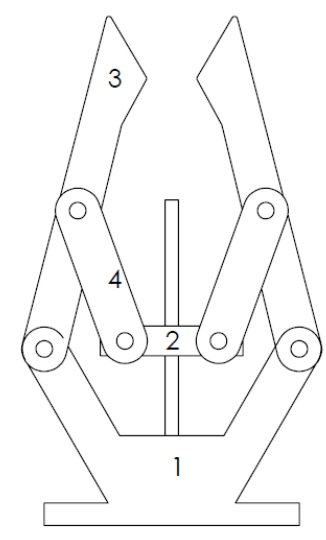

Fig. 1. Efector final

\subsection{Cinemática del mecanismo}

En la Figura 2 se observa un diagrama esquemático del mecanismo del efector final, donde cada vector $r_{i}$ está relacionado con el i-ésimo eslabón del mecanismo. Del mecanismo propuesto se establece la ecuación de cierre de circuito como:

$$
\boldsymbol{r}_{1}+\boldsymbol{r}_{3}=\boldsymbol{r}_{2}+\boldsymbol{r}_{0}+\boldsymbol{r}_{4}
$$

Aplicando notación polar a cada término de (1), se obtiene:

$$
r_{1} e^{j \theta_{1}}+r_{3} e^{j \theta_{3}}=r_{2} e^{j \theta_{2}}+r_{0} e^{j \theta_{0}}+r_{4} e^{j \theta_{4}}
$$

Usando la ecuación de Euler en (2) y separando las partes real e imaginaria:

$$
\begin{aligned}
& r_{1} \cos \theta_{1}+r_{3} \cos \theta_{3}=-r_{0}+r_{4} \cos \theta_{4} \\
& r_{1} \sin \theta_{1}+r_{3} \sin \theta_{3}=r_{2}+r_{4} \sin \theta_{4}
\end{aligned}
$$

Para obtener la posición angular $\theta_{3}$, el lado izquierdo del sistema de ecuaciones (3) se expresa en términos de $\theta_{4}$ :

$$
\begin{aligned}
& r_{4} \cos \theta_{4}=r_{0}+r_{1} \cos \theta_{1}+r_{3} \cos \theta_{3} \\
& r_{4} \sin \theta_{4}=r_{1} \sin \theta_{1}+r_{3} \sin \theta_{3}-r_{2}
\end{aligned}
$$


Eric Santiago-Valentín, Adrián Solano-Palma, Pedro Bautista-Camino, et al.

Elevando al cuadrado (4) y sumando sus términos se obtiene la ecuación de Freudenstein en su forma compacta [10], la cual se establece como:

$$
A \cos \theta_{3}+B \sin \theta_{3}+C=0
$$

donde:

$$
\begin{aligned}
& A=2 r_{3}\left(r_{0}+r_{1} \cos \theta_{1}\right) \\
& B=2 r_{3}\left(r_{1} \sin \theta_{1}-r_{2}\right) \\
& C=r_{0}^{2}+r_{1}^{2}+r_{2}^{2}+r_{3}^{2}-r_{4}^{2}+2 r_{0} r_{1} \cos \theta_{1}-2 r_{1} r_{2} \sin \theta_{1}
\end{aligned}
$$

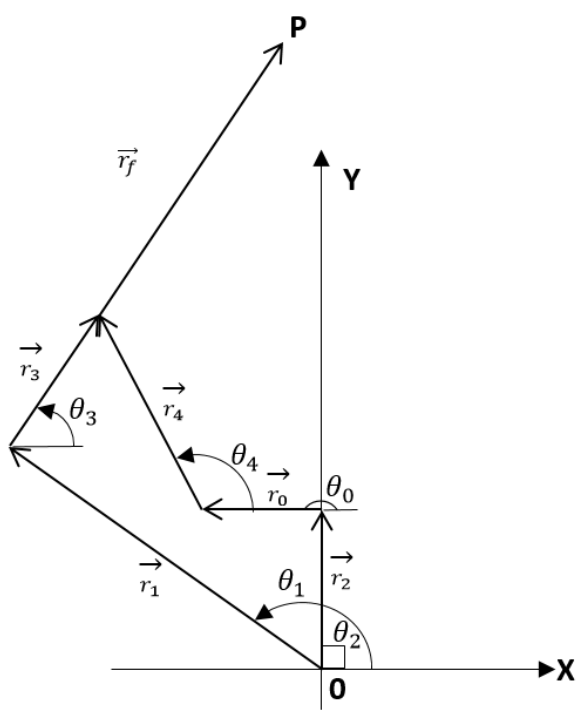

Fig. 2. Diagrama esquemático del efector final

El ángulo $\theta_{3}$ puede ser calculado como una función de los parámetros $A, B$ y $C$. Dicha solución puede ser obtenida al expresar $\sin \theta_{3}$ y $\cos \theta_{3}$ en términos de $\tan \left(\theta_{3} / 2\right)$ como sigue:

$$
\sin \theta_{3}=\frac{2 \tan \left(\theta_{3} / 2\right)}{1+\tan ^{2}\left(\theta_{3} / 2\right)}, \cos \theta_{3}=\frac{1-\tan ^{2}\left(\theta_{3} / 2\right)}{1+\tan ^{2}\left(\theta_{3} / 2\right)}
$$

sustituyendo éstas en (5), se obtiene una ecuación lineal de segundo orden:

$$
[C-A] \tan ^{2}\left(\theta_{3} / 2\right)+[2 B] \tan \left(\theta_{3} / 2\right)+A+C=0
$$

Resolviendo (10), la posición angular $\theta_{3}$ esta dada por (11).

$$
\theta_{3}=2 \arctan \left[\frac{-B \pm \sqrt{B^{2}+A^{2}-C^{2}}}{C-A}\right]
$$


Un procedimiento similar al anterior se lleva a cabo para obtener $\theta_{4}$; a partir de (3) se llega a la ecuación de Freudenstein en su forma compacta:

$$
D \cos \theta_{4}+E \sin \theta_{4}+F=0
$$

donde:

$$
\begin{aligned}
& D=-2 r_{4}\left(r_{0}+r_{1} \cos \theta_{1}\right) \\
& E=2 r_{4}\left(r_{2}-r_{1} \sin \theta_{1}\right) \\
& F=r_{0}^{2}+r_{1}^{2}+r_{2}^{2}+r_{4}^{2}-r_{3}^{2}+2 r_{0} r_{1} \cos \theta_{1}-2 r_{1} r_{2} \sin \theta_{1}
\end{aligned}
$$

Por lo tanto, la posición angular $\theta_{4}$ puede calcularse como:

$$
\theta_{4}=2 \arctan \left[\frac{-E \pm \sqrt{D^{2}+E^{2}-F^{2}}}{F-D}\right]
$$

Finalmente, la posición de los extremos del efector final $P$ esta dada por:

$$
\begin{aligned}
& P_{x}=r_{1} \cos \theta_{1}+r_{f} \cos \theta_{3} \\
& P_{y}=r_{1} \sin \theta_{1}+r_{f} \sin \theta_{3}
\end{aligned}
$$

\subsection{Análisis de fuerzas en el efector final}

Como se ha mencionado previamente, uno de los aspectos más importantes al diseñar un efector final tiene que ver con la fuerza de agarre o con la transmisión de la misma. La Figura 3 muestra la distribución de fuerzas en los elementos mecánicos del efector, donde $P$ representa la fuerza de entrada o impulsión del sistema, $F_{T}$ es la fuerza de agarre ejercida por el efector sobre el objeto de interés y $F_{k j}$ representa la fuerza que ejerce el k-ésimo elemento sobre el j-ésimo. Como se puede observar:

$$
\begin{aligned}
F_{42 i} & =F_{42} \\
F_{24} & =F_{34} \\
F_{34} & =\frac{P}{2 \sin \theta} \\
\theta & =\pi-\theta_{4}
\end{aligned}
$$

Así mismo, tomando el momento de fuerza respecto del punto $A$ y considerando $\sum M=0$ :

$$
F_{T}=\frac{r_{3} \sin \left(\theta_{4}-\theta_{3}\right)}{2 r_{f} \sin \theta_{3} \sin \theta_{4}} P
$$

\section{Estrategias de optimización}

Una vez que se han establecido apropiadamente la cinemática y la dependencia de la fuerza con las relaciones geométricas del mecanismo, el problema de diseño se puede definir como un problema de optimización numérica, por lo que se requiere especificar tanto las relaciones matemáticas que permitan evaluar el desempeño del sistema como las restricciones a los que estará sujeto. 

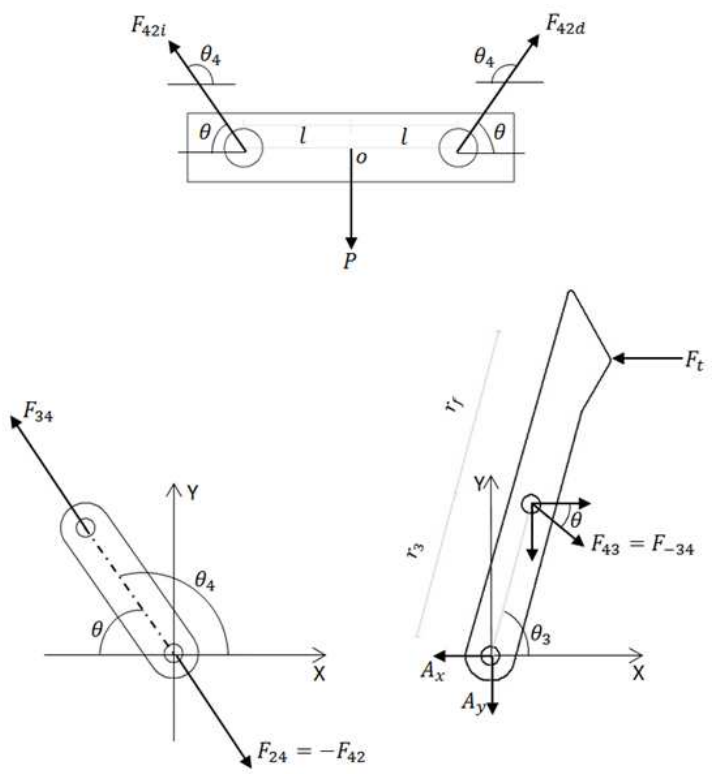

Fig. 3. Diagrama de la distribución de fuerzas en el efector final

\subsection{Función objetivo}

Un criterio para cuantificar el desempeño del efector final considera que la fuerza de sujeción debe ser constante en todo su espacio de trabajo. Como se vió en la sección anterior, la posición del extremo del efector depende de la tuerca en el tornillo de potencia, requiriéndose que la fuerza en los extremos de máximo y mínimo desplazamiento sea constante. Proponiendo la función objetivo de (24), ésta debe tener un valor mínimo para asegurar una fuerza casi constante en todo el espacio de trabajo; así, el valor ideal de la función es cero.

$$
f\left(r_{2}\right)=\left(F_{T}\left(r_{2 \min }\right)-F_{T}\left(r_{2 \max }\right)\right)^{2}
$$

Finalmente, es necesario definir el espacio de trabajo del efector final, para lo cual se tiene que:

$$
\begin{aligned}
& X_{\min }: \text { Dimensión mínima del objeto de interés. } \\
& X_{\max } \text { :Dimensión máxima del objeto de interés. } \\
& X_{G}: \text { Rango máximo de desplazamiento de los extremos del efector. } \\
& r_{2 m i n}: \text { Valor mínimo de la posición de la tuerca. } \\
& r_{2 m a x}: \text { Valor máximo de la posición de la tuerca. } \\
& R_{2 m a x}: \text { Rango de variación de la posición de la tuerca. }
\end{aligned}
$$

\subsection{Restricciones de diseño}

Debido a que el efector final es la interfaz entre un sistema automatizado y el mundo real, es necesario que la interacción entre ambos dominios sea correcta. En 
este sentido, se debe asegurar la sujeción apropiada para las posiciones mínima y máxima del espacio de trabajo, es decir:

$$
\begin{aligned}
& P_{x}\left(r_{2 \min }\right) \leq X_{\min } \\
& P_{x}\left(r_{2 \min }\right) \geq 0 \\
& P_{x}\left(r_{2 \max }\right) \geq X_{\max } \\
& P_{x}\left(r_{2 \min }\right) \leq X_{G}
\end{aligned}
$$

Considerando una geometría esférica para el objeto a asir, se debe cumplir que la coordenada $P_{y}$ del extremo del efector sea mayor que la dimensión máxima del objeto más el rango de variación del tornillo de potencia y la tuerca, esto es:

$$
P_{y}\left(r_{2 \max }\right) \geq X_{\max }+R_{2 \max }
$$

\section{Diseño óptimo del mecanismo}

Como se mencionó, en este trabajo se lleva a cabo el diseño óptimo para transmisión de fuerza de un efector final. Para ello se parametrizó el sistema, ya que una descripción apropiada de las variables permite al diseñador una amplia posibilidad de reconfiguración del sistema.

\subsection{Variables de diseño}

Sea el vector de variables de diseño para el efector final, establecido como:

$$
\begin{aligned}
\boldsymbol{p} & =\left[p_{1}, p_{2}, p_{3}, p_{4}, p_{5}, p_{6}, p_{7}, p_{8}\right]^{T} \\
& =\left[r_{1}, r_{2 \min }, r_{2 \max }, r_{3}, r_{4}, r_{0}, r_{f}, \theta_{1}\right]^{T}
\end{aligned}
$$

donde las variables $r_{1}, r_{3}, r_{4}, r_{0}, r_{f}$ corresponden a las longitudes de las barras del mecanismo, $r_{2 \min }$ y $r_{2 \max }$ a las posiciones de la tuerca en la mínima y máxima apertura y finalmente $\theta_{1}$ al ángulo del extremo de la pieza base respecto del origen del sistema coordenado.

\subsection{Problema de optimización}

Sea el problema de optimización numérica mono-objetivo descrito por (32) hasta (46), para obtener la solución al problema de diseño de síntesis para la transmisión óptima de fuerza del efector final:

$$
\begin{aligned}
& \operatorname{Min} f(\boldsymbol{p})=\left[F_{T}\left(r_{2 \min }\right)-F_{T}\left(r_{2 \max }\right)\right]^{2} \\
& \boldsymbol{p} \in \mathbb{R}^{8}
\end{aligned}
$$

con las cotas:

$$
\begin{aligned}
0 & \leq p_{i} \leq 150, \quad i=1,4,5,6,7 \\
0 & \leq p_{i} \leq R_{2 \max }, \quad i=2,3 \\
\frac{\pi}{2} & \leq p_{i} \leq \pi, \quad i=8
\end{aligned}
$$


sujeto a:

$$
\begin{aligned}
& g_{1}(\boldsymbol{p})=\left|P_{x}\left(r_{2 \min }\right)\right|-X_{\min } \leq 0 \\
& g_{2}(\boldsymbol{p})=-\left|P_{x}\left(r_{2 \min }\right)\right| \leq 0 \\
& g_{3}(\boldsymbol{p})=\left|P_{x}\left(r_{2 \max }\right)\right|-X_{G} \leq 0 \\
& g_{4}(\boldsymbol{p})=X_{\max }-\left|P_{x}\left(r_{2 \max }\right)\right| \leq 0 \\
& g_{5}(\boldsymbol{p})=X_{\max }+R_{2 \max }-P_{y}\left(r_{2 \max }\right) \leq 0 \\
& g_{6}(\boldsymbol{p})=p_{2}-p_{3} \leq 0 \\
& g_{7}(\boldsymbol{p})=p_{4}-p_{7} \leq 0
\end{aligned}
$$

y con el espacio de trabajo:

$$
\begin{aligned}
X_{\min } & =20 \\
X_{\max } & =150 \\
X_{G} & =200 \\
R_{2 \max } & =50
\end{aligned}
$$

\section{Algoritmo de optimización}

El algoritmo de Evolución Diferencial (ED) es una de las técnicas metaheurísticas más populares, y se ha aplicado para resolver diversos problemas no lineales, no diferenciables y multimodales [11]. Dicho algoritmo toma una población inicial aleatoria de soluciones, y en cada generación se producen nuevos individuos candidatos aplicando operadores de reproducción (cruza y mutación). La aptitud de cada descendiente se evalúa para que compita con el individuo padre, y así determinar cuál de ellos se conservará para la generación siguiente. Una de las principales ventajas de la ED es su número de parámetros de control, ya que solamente se requieren tres parámetros de entrada para controlar el proceso de búsqueda; esto es, el tamaño de la población $N P$, la constante de diferenciación $F$ que controla la amplificación de la variación diferencial y el parámetro de control de cruza $C R$ [12]. Las características generales de esta técnica son:

- Representación de soluciones como individuos

- Selección de padres

- Recombinación o cruza

- Mutación

- Selección de sobrevivientes y variantes

El pseudocódigo correspondiente a la ED se muestra en el Algoritmo 5.1; ahora bien, en la etapa de competencia para la sustitución generacional se utilizan las reglas de factibilidad de Deb [13], las cuales se listan a continuación:

1. Entre dos individuos factibles, se escoge al de mejor función objetivo.

2. Entre dos individuos no factibles, se escoge al que tenga un valor menor en la suma de violaciones a las restricciones. 
3. Entre un individuo factible y otro no factible, se escoge al factible.

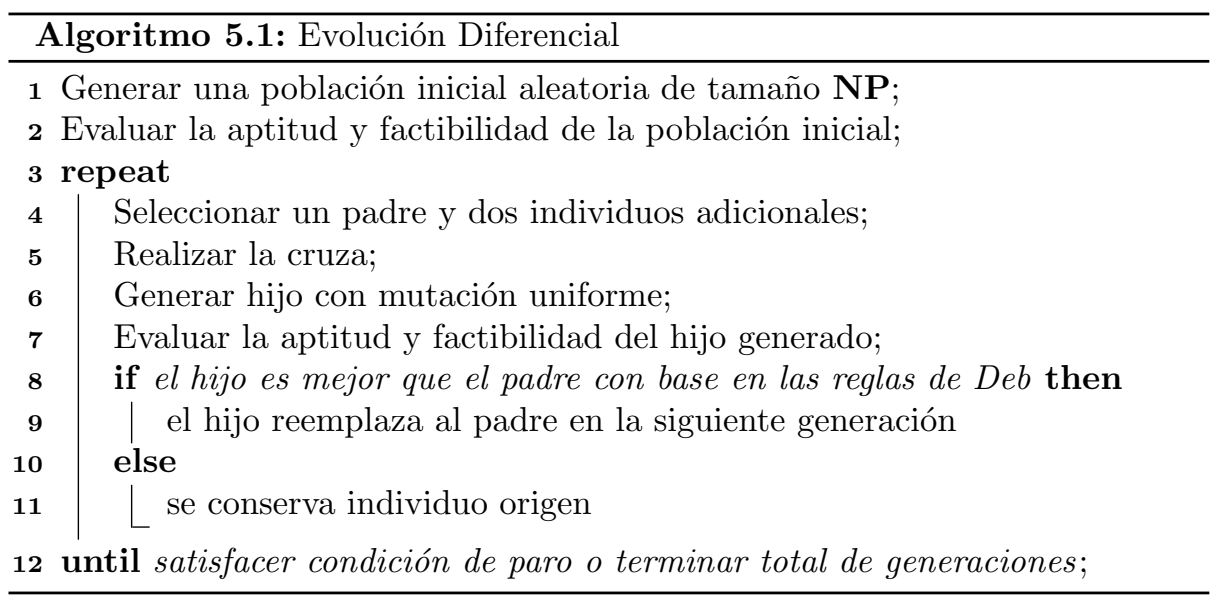

\subsection{Implementación computacional}

La implementación del algoritmo se realizó en Matlab R2013aß, sobre una plataforma computacional con las siguientes características: procesador Intel Core i7 @2.2GHz, con 8GB de memoria RAM y sistema operativo Windows 8 .

En el programa se implementó un módulo para el cálculo de la función objetivo y las restricciones, con el fin de evaluar la aptitud de los individuos. La factibilidad se deduce con base en el cálculo de la suma de violación de restricciones (SVR); dicho valor indica si el individuo se encuentra en la zona factible [13]. Si las variables del vector de diseño cumplen con las restricciones se calcula la función objetivo; en caso contrario a ésta se le asigna un valor grande como penalización para fines de ahorro computacional $(\mathrm{FO}=1000)$. Así mismo, las características del problema se muestran en el Cuadro 1, donde $l i$ es el número de restricciones de desigualdad lineales, $n i$ son las restricciones de desigualdad no lineales, le las restricciones de igualdad lineales, y ne las restricciones de igualdad no lineales.

Tabla 1. Características del problema

\begin{tabular}{|l|l|l|l|l|l|l|}
\hline Max-Eval & N & Tipo Función & $l i$ & $n i$ & $l e$ & $n e$ \\
\hline 30,000 & 8 & No Separable & 7 & 0 & 0 & 2 \\
\hline
\end{tabular}

En las simulaciones se comprobó que el tamaño de la población (NP) y número de generaciones (GMAX) son los parámetros más importantes para sintonizar el rendimiento del algoritmo. En este trabajo se utiliza como condición 
de paro el número de evaluaciones (EVAL) en lugar del número de generaciones, para efectos de futuras comparaciones contra otros métodos de optimización.

\section{Resultados}

Después de algunas pruebas preliminares para la calibración del algoritmo se realizó un conjunto de treinta simulaciones, cuyos mejores resultados se muestran en el Cuadro 3. Los parámetros empleados fueron: tamaño de población $N P=16$, número de evaluaciones $E V A L=30,000$, factor de cruza $C R=[0.8,1.0]$ por ejecución, y factor de escala $F=[0.3,0.9]$ por generación; estos últimos dos parámetros se calcularon de forma aleatoria entre los rangos mencionados. Se puede observar que la mayoría de las simulaciones alcanzaron el valor ideal de la función objetivo $(\mathrm{FO}=0)$ con respecto a lo planteado en la Sección 3.1, mientras que en los últimos dos casos se obtuvieron valores muy cercanos al valor óptimo. Desde el punto de vista algorítmico estos tambien se consideran casos de éxito, ya que ambos valores se encuentran dentro de la tolerancia establecida entre el óptimo conocido y el resultado, que en este caso fue de $1 \times 10^{-4}$.

Como un dato relevante se puede apreciar que el problema es multimodal, ya que se obtuvieron diferentes vectores de diseño con el mismo valor óptimo de la función objetivo. Por lo tanto, para esta clase de problemas el enfoque de la sintonización del algoritmo no debe centrarse en la exploración de la zona factible sino en la explotación de la misma; esto es, no es necesario utilizar un conjunto grande de individuos en la configuración inicial ya que con pocas soluciones se realiza una buena explotación de la zona factible con menos generaciones.

Tabla 2. Estadísticas de las simulaciones numéricas

\begin{tabular}{|c|c|}
\hline Mejor & 0 \\
\hline Mediana & 0 \\
\hline Peor & $1.316873 \mathrm{E}-06$ \\
\hline Promedio & $4.389577 \mathrm{E}-08$ \\
\hline Desviación estándar & $2.363859 \mathrm{E}-07$ \\
\hline
\end{tabular}

Los resultados del análisis estadístico correspondiente a las treinta simulaciones se incluyen en el Cuadro 2, donde se observa la solidez de las las soluciones obtenidas. Como se mencionó anteriormente se trata de un problema multimodal ya que la mayoría de las soluciones cumplen con el valor óptimo ideal de la función objetivo $(\mathrm{FO}=0)$, con la ventaja de que dichas soluciones producen diferentes configuraciones del mecanismo. Esto se muestra en la Figura 4 , en donde se aprecia el modelado de tres diferentes configuraciones de diseño tomadas del Cuadro 3, dichos modelos se realizaron con SolidWorks 2013®.

En la Figura 5 se muestra el comportamiento de la función objetivo en tres casos distintos; únicamente se incluye la parte esencial de la gráfica por razones 


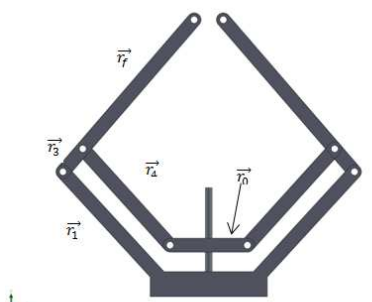

a) Barra $\overrightarrow{r_{0}}$ con mayor tamaño

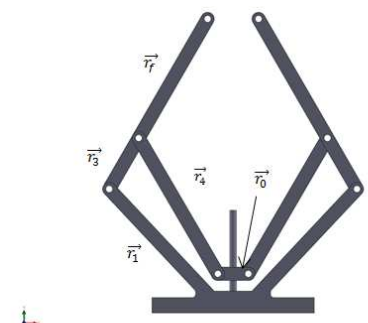

b) Barra $\overrightarrow{r_{3}}$ con mayor tamaño

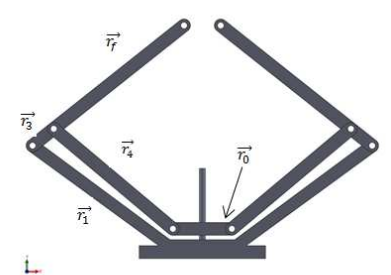

c) Barra $\overrightarrow{r_{1}}$ con mayor tamaño

Fig. 4. Modelado de tres configuraciones distintas del efector final

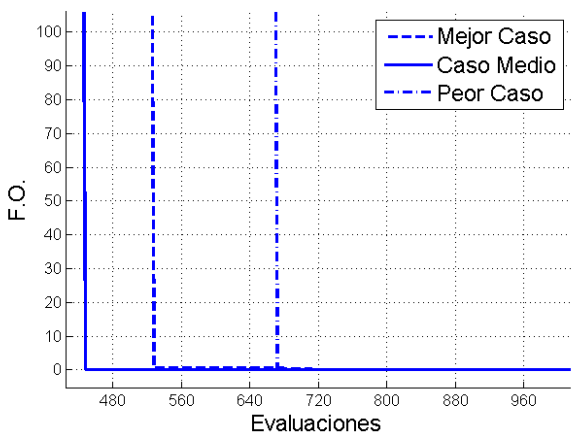

Fig. 5. Convergencia de la función objetivo hacia el valor óptimo

de visualización. Para el mejor caso se tomó la primera simulación del Cuadro 3, mientras que para los casos medio y peor se consideraron las dos soluciones con función objetivo diferente a cero. Considerando que las simulaciones se llevaron sobre 30,000 evaluaciones, se observa que en todos los casos el algortimo presenta una convergencia muy rápida, entre las 400 y 650 evaluaciones. En la Figura 6 se muestra el comportamiento de los individuos que entran a la zona factible para los tres casos ya descritos; como se observa, nuevamente el algortimo presenta un comportamiento muy estable ya que en todas las soluciones la totalidad de individuos entró a las zona factible después de un número relativamente corto de evaluaciones de la función objetivo $(<1500)$.

\section{Conclusiones}

En este trabajo se presenta una solución novedosa para la síntesis dimensional de un efector final, con transferencia constante de fuerza en todo el espacio de trabajo del mecanismo; dicha síntesis se llevó a cabo proponiendo un problema de optimización numérica el cual se resolvió utilizando el algoritmo de ED. Para este 
Tabla 3. Mejores vectores de solución por cada simulación

\begin{tabular}{|c|c|c|c|c|c|c|c|c|c|}
\hline $\mathbf{N}$ & $r_{1}$ & $r_{2 \min }$ & $r_{2 \max }$ & $r_{3}$ & $r_{4}$ & $r_{0}$ & $r_{f}$ & $\theta_{1}$ & F.O. \\
\hline 1 & 125.6648 & 14.8219 & 49.8588 & 31.8815 & 78.1777 & 52.1401 & 148.8865 & 2.6537 & 0 \\
\hline 2 & 132.1698 & 4.01253 & 48.0439 & 33.3737 & 110.0118 & 25.7951 & 149.6869 & 2.6122 & 0 \\
\hline 3 & 110.2324 & 0.5953 & 48.3966 & 66.5903 & 132.3761 & 9.2966 & 149.7525 & 2.5073 & 0 \\
\hline 4 & 123.4283 & 10.8431 & 48.5606 & 26.1936 & 107.3209 & 14.7612 & 146.0794 & 2.5258 & 0 \\
\hline 5 & 111.9395 & 8.5666 & 49.8989 & 27.6270 & 102.0167 & 8.1545 & 146.0073 & 2.4823 & 0 \\
\hline 6 & 118.4443 & 0.2539 & 49.9138 & 54.0889 & 122.0082 & 18.2222 & 149.8627 & 2.5485 & 0 \\
\hline 7 & 108.3848 & 14.6305 & 47.4309 & 36.8179 & 102.9599 & 13.5255 & 149.8074 & 2.4559 & 0 \\
\hline 8 & 109.7232 & 0.1354 & 48.9268 & 69.2182 & 142.7452 & 0.2063 & 149.6518 & 2.4521 & 0 \\
\hline 9 & 117.4040 & 12.6399 & 47.0143 & 27.3896 & 111.9544 & 3.7957 & 149.5433 & 2.4668 & 0 \\
\hline 10 & 111.1112 & 0.8470 & 49.8789 & 63.8318 & 136.8610 & 3.5517 & 149.6605 & 2.4586 & 0 \\
\hline 11 & 104.6851 & 16.0943 & 49.2388 & 41.6483 & 109.7281 & 4.2353 & 149.9761 & 2.3857 & 0 \\
\hline 12 & 119.6796 & 2.7717 & 42.1311 & 31.0122 & 120.6785 & 3.1324 & 148.6101 & 2.5256 & 0 \\
\hline 13 & 143.9719 & 19.2837 & 47.6935 & 16.9678 & 61.93212 & 79.0936 & 149.6438 & 2.7446 & 0 \\
\hline 14 & 137.6210 & 5.3280 & 48.3020 & 23.0749 & 118.8039 & 14.7306 & 144.3349 & 2.5958 & 0 \\
\hline 15 & 130.8984 & 13.9086 & 49.6894 & 20.3562 & 100.1112 & 26.0418 & 148.8370 & 2.5755 & 0 \\
\hline 16 & 146.6675 & 19.3563 & 49.8179 & 14.4672 & 130.6670 & 2.8650 & 149.8476 & 2.5628 & 0 \\
\hline 17 & 125.8573 & 2.7284 & 49.7670 & 47.4768 & 110.2369 & 30.0987 & 149.7088 & 2.6155 & 0 \\
\hline 18 & 118.2962 & 0.9036 & 49.5427 & 50.5869 & 130.9261 & 2.5862 & 149.4477 & 2.4894 & 0 \\
\hline 19 & 145.1526 & 20.3894 & 49.1449 & 12.0728 & 116.7860 & 17.3613 & 149.7476 & 2.5925 & 0 \\
\hline 20 & 148.4874 & 0.0173 & 45.2371 & 25.3724 & 141.7813 & 2.0523 & 149.8704 & 2.6161 & 0 \\
\hline 21 & 126.0129 & 1.1487 & 48.9555 & 41.8592 & 100.4658 & 35.3734 & 149.8708 & 2.6562 & 0 \\
\hline 22 & 123.3468 & 0.1855 & 49.3994 & 43.7044 & 131.2199 & 4.2993 & 148.1329 & 2.4976 & 0 \\
\hline 23 & 107.0277 & 0.6777 & 49.9972 & 81.8748 & 147.6265 & 3.7487 & 149.8855 & 2.4635 & 0 \\
\hline 24 & 114.3837 & 4.0352 & 49.6333 & 45.3223 & 123.3099 & 4.8782 & 149.9987 & 2.4612 & 0 \\
\hline 25 & 124.6722 & 3.4510 & 49.6703 & 52.9403 & 101.1188 & 42.5436 & 149.7304 & 2.6740 & 0 \\
\hline 26 & 126.9049 & 10.5596 & 49.9930 & 35.4906 & 81.6281 & 52.5143 & 149.3339 & 2.6739 & 0 \\
\hline 27 & 118.0669 & 13.4724 & 48.6274 & 34.4630 & 100.2700 & 23.3688 & 149.2731 & 2.5284 & 0 \\
\hline 28 & 113.7514 & 6.6159 & 49.8117 & 56.4621 & 115.5941 & 21.4816 & 149.3870 & 2.5377 & 0 \\
\hline 29 & 130.8298 & 3.8528 & 5.93549 & 0.8488 & 104.9226 & 33.7778 & 149.9932 & 2.3785 & $1.61 \mathrm{E}-19$ \\
\hline 30 & 119.3823 & 35.8580 & 40.5029 & 1.9082 & 38.2731 & 75.0968 & 150 & 2.5201 & $.31 \mathrm{E}-06$ \\
\hline
\end{tabular}




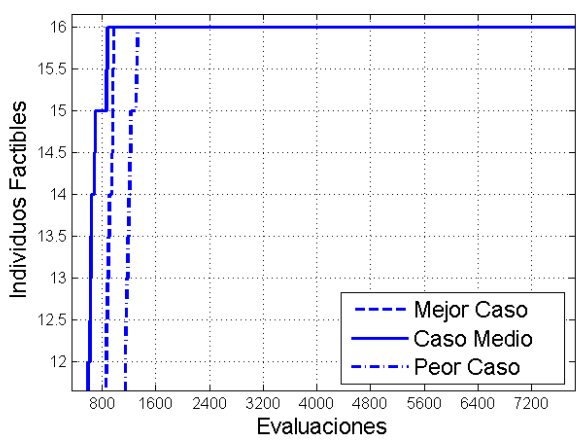

Fig. 6. Individuos factibles por evaluaciones

caso en particular se observó que, de acuerdo a los resultados obtenidos, existen varias soluciones que satisfacen el valor óptimo ideal de la función objetivo $(\mathrm{FO}=0)$, por lo que se puede concluir que es un problema de caracter multimodal. En este sentido, el algoritmo realiza una buena exploración y posterior explotación de la zona factible, para encontrar diversas soluciones óptimas; dichas soluciones representan diferentes configuraciones del mecanismo, que deben ser posteriormente analizadas desde el punto de vista de ingeniería para valorar si cumplen tanto con las restricciones de fabricación como de carácter estético. Lo anterior debido a que no todas las soluciones son físicamente construibles dadas las limitaciones de tamaño de algunos elementos, la cantidad de material que se emplearía para su construcción o la resistencia derivada de su tamaño y posición en el mecanismo, por mencionar algunos ejemplos.

Finalmente, se concluye que el uso de algoritmos evolutivos aplicados a problemas reales de ingeniería presenta un alto grado de confiabilidad y desempeño, siempre y cuando se haya realizado una sintonización adecuada para cada uno de los problemas a resolver. Así, esta clase de algoritmos presentan una opción viable de solución a problemas de optimización duros del mundo real, diferentes en su concepción a los problemas de benchmark con que usualmente se prueban estas técnicas de optimización; esto es, representan una herramienta de gran utilidad para problemas cuya solución no se conoce previamente.

Agradecimientos. Todos los autores agradecen el apoyo del Instituto Politécnico Nacional a través de la SIP vía el proyecto SIP-20151320. El primer autor agradece al CONACyT por la beca para estudios de posgrado en el CIDETECIPN.

\section{Referencias}

1. Sam, R. and Nefti, S.: Design and Development of Flexible Robotic Gripper for Handling Food Products. 10th Intl. Conf. on Control, Automation, Robotics and Vision, IEEE, 1689-1684, Hanoi, Vietnam, (2008) 
2. Storn, R. and Price, K.: An Introduction to Differential Evolution, New Ideas in Optimization Differential Evolution - A simple and efficient adaptive scheme for global optimization over continuous spaces, )International Computer Science Institute, Muenchen, Germany (1995)

3. Osyczka, A., Krenich and Kara ,J.; Evolutionary multi criteria design optimization of robot grippers. Department of Mechanical Engineering, Cracow University of Technology, Polonia, (1999)

4. Cuadrado, J., Naya, M.A., Ceccarelli, M. and Carbone, G.: An Optimum Design Procedure for Two-Finger Grippers: A Case of Study. Universidad de La Coruña, España, (2001)

5. Saravanan, R., Ramabalan, S., Godwin Raja Ebenezer, N. and Dharmaraja, C.: Evolutionary multi criteria design optimization of robot grippers. Applied Soft Computing, Elsevier B.V., 159-172, Tamil Nadu, India, (2009)

6. Moses Mullar, S. and Satya Meher, R.: Optimizing of Robot Gripper Configurations Using Ant Colony Optimization. International Journal of Engineering Research and Technology, IJERT, 2655-2662, Andhra Pradesh, India, (2013)

7. Portilla-Flores E. A., Santiago-Valentin E., Solano Palma A., Vega Alvarado, E. y Calva Yáñez, M. B.: Cálculo de fuerza para agarre óptimo de un efector final de tres dedos utilizando el algoritmo de forrajeo de bacterias. Second International Conference on Advanced Mechatronics, Design, and Manufacturing Technology, 65-70, Colombia, (2014)

8. Chica Leal, A., Prada Jiménez, V., Rey Hernández, H. y Leon Hidalgo, C.: Diseño y construcción de un gripper implementando optimización. Second International Conference on Advanced Mechatronics, Design, and Manufacturing Technology, 219-222, Colombia, (2014)

9. Lanni, Ch., and Ceccarelli, M.: An Optimization Problem Algorithm for Kinematic Design of Mechanisms for Two-Finger Grippers. The Open Mechanical Engineering Journal, 49-62, Colombia, (2009)

10. Shigley J.E. y Uicker J.J. Jr.: Teoría de Máquinas y Mecanismos, McGraw Hill, México (1988)

11. Price K.V.: An Introduction to Differential Evolution, New Ideas in Optimization, Mc Graw Hill, UK (1999)

12. Boussaid, I., Lepagnot,J. and Siarry, P.: A Survey on Optimization Metaheuristics. Information Sciences. 237, 82-117 (2013)

13. Deb, K.: An Efficient Constraint Handling Method for Genetic Algorithms. Computer Methods in Applied Mechanics and Engineering. 186, 311-338 (2000) 